\title{
Transcriptomic and proteomic analysis of human hepatic stellate cells treated with natural taurine
}

\author{
JIAN LIANG, XIN DENG, FA-SHENG WU and YAN-FANG TANG \\ Ruikang Hospital of Guangxi Traditional Chinese Medical University, Nanning, Guangxi 530011, P.R. China
}

Received September 24, 2012; Accepted February 14, 2013

DOI: $10.3892 / \mathrm{mmr} .2013 .1389$

\begin{abstract}
The aim of this study was to investigate the differential expression of genes and proteins between natural taurine (NTau)-treated hepatic stellate cells (HSCs) and control cells as well as the underlying mechanism of NTau in inhibiting hepatic fibrosis. A microculture tetrazolium (MTT) assay was used to analyze the proliferation of NTau-treated HSCs. Flow cytometry was performed to compare the apoptosis rate between NTau-treated and non-treated HSCs. Proteomic analysis using a combination of 2-dimensional gel electrophoresis (2DE) and mass spectrometry (MS) was conducted to identify the differentially expressed proteins. Microarray analysis was performed to investigate the differential expression of genes and real-time polymerase chain reaction (PCR) was used to validate the results. The experimental findings obtained demonstrated that NTau decreased HSC proliferation, resulting in an increased number of cells in the G0/G1 phase and a reduced number of cells in the $\mathrm{S}$ phase. Flow cytometric analysis showed that NTautreated HSCs had a significantly increased rate of apoptosis when compared with the non-treated control group. A total of 15 differentially expressed proteins and 658 differentially expressed genes were identified by 2DE and MS, and microarray analysis, respectively. Gene ontology (GO) functional analysis indicated that these genes and proteins were enriched in the function clusters and pathways related to cell proliferation, cellular apoptosis and oxidation. The transcriptome and proteome analyses of NTau-treated HSCs demonstrated that NTau is able to significantly inhibit cell proliferation and promote cell apoptosis, highlighting its potential therapeutic benefits in the treatment of hepatic fibrosis.
\end{abstract}

Correspondence to: Professor Xin Deng, Ruikang Hospital of Guangxi Traditional Chinese Medical University, 10 Huadong Road, Nanning, Guangxi 530011, P.R. China

E-mail: dx8848@126.com

Key words: hepatic fibrosis, taurine, hepatic stellate cell, microarray, cell proliferation, cell cycle, apoptosis

\section{Introduction}

Hepatic fibrosis refers to the excessive accumulation of extracellular matrix (ECM) components in the liver. It occurs in most types of chronic liver diseases, including liver cirrhosis, liver failure, and portal hypertension, and often requires liver transplantation (1). The activation of hepatic stellate cells (HSCs) is an important event by which this cell type, which is otherwise quiescent, expresses $\alpha$-smooth muscle actin $(\alpha$-SMA), assumes a myofibroblastic phenotype and synthesizes fibrillar collagens $(2,3)$. Therefore, the inhibition of HSC proliferation, the regulation of the HSC cell cycle, and the facilitation of HSC apoptosis are important therapeutic approaches for hepatic fibrosis-related liver diseases.

Taurine (2-aminoethanesulfonic acid) is an organic acid which is abundant in the human body. Natural taurine (NTau) has emerged as an alternative candidate for therapeutic intervention since it is effective in preventing hepatic fibrosis and reducing cirrhosis $(4,5)$. Supplementation with exogenous taurine is able to extensively inhibit the deposition of ECM and mitigate the degree of hepatic fibrosis (2). Several studies have focused on the specific gene regulation associated with the protective effect of taurine against hepatic damage (6-8), but the genome-wide genes, proteins and functional pathways underlying the hepatic protection have yet to be fully elucidated.

Gene-expression profiling through microarray analysis may shed light on useful clues to the taurine-mediated gene regulation. Furthermore, a proteomics approach may also be used to elucidate global protein expression and facilitate the discovery of potential drug targets. However, studies using microarray or proteomic technologies to investigate the molecular mechanism of taurine treatment for liver diseases have not been previously been conducted. Therefore, an integrative analysis of transcriptome and proteome levels was designed to illuminate the changes of gene and protein expression in human HSCs treated with NTau.

\section{Materials and methods}

NTau extraction. NTau (2-aminoethanesulfonic acid) was extracted from black clams (Meretrix meretrix L.). Briefly, the clam meats were weighed and minced in an electrical blender (4000 rpm), for $\sim 10 \mathrm{sec}$. The mince was further homogenized for 30 min after adding distilled water (1 liter). The mixture was boiled in water for $30 \mathrm{~min}$, followed by filtering through 
4 layers of gauze. The residue on the top of the gauze was discarded, and the filtrate was then centrifuged $(3000 \mathrm{rpm})$ to obtain the supernatant, which was then de-acidified with $\mathrm{HCl}$ $\left(\mathrm{HCl}: \mathrm{H}_{2} \mathrm{O}=3\right)$. After centrifuging, the proteins were adjusted to a $\mathrm{pH}$ of 10 with a $\mathrm{NaOH}(20 \%)$ aqueous solution to yield the de-alkalinated protein. Following adjustment of the $\mathrm{pH}$ value to 5 , the supernatant was further condensed. The other unwanted amino acids and pigments were removed by column chromatography using strong-acid cation-exchange resin as the solid phase and eluting with distilled water. The resultant NTau was quantitatively measured by high-performance liquid chromatography (HPLC), and the purity of the NTau was determined to be $98.8 \%$.

Cell culture. LX-2 human HSCs (purchased from the Cell Bank at Xiangya Central Experiment Laboratory of Central South University, Changsha, China) were cultivated at $37^{\circ} \mathrm{C}$ in Dulbecco's modified Eagle's medium (DMEM; Gibco-BRL, Carlsbad, CA, USA), and were supplemented with $10 \%$ fetal bovine serum (FBS) and penicillin-streptomycin in a $5 \% \mathrm{CO}_{2}$ humidified incubator. Cells $\left(1 \times 10^{4}\right.$ or $\left.5 \times 10^{5}\right)$ were seeded in 96-well plates or $35-\mathrm{mm}$ dishes. The cells were cultured in serum-free medium for $24 \mathrm{~h}$ and then treated with $40 \mathrm{mM}$ NTau for $48 \mathrm{~h}$.

Cell proliferation analysis. The proliferation activity of the LX-2 cells was measured by a microculture tetrazolium (MTT) colorimetric assay. LX-2 cells $\left(1 \times 10^{4}\right.$ cells $\left./ \mathrm{ml}\right)$ were cultured in 96-well plates in DMEM with $10 \%$ FBS medium and then tranferred to serum-free medium for an additional $24 \mathrm{~h}$, and triplicate wells of cells were incubated for $48 \mathrm{~h}$ in the presence 0-50 mM NTau. Cells in the various treatment groups were then incubated with MTT [5 $\mathrm{mg} / \mathrm{ml}$ in phosphate-buffered solution (PBS)] for $4 \mathrm{~h}$ before harvesting. The optical density was measured using an ELISA reader at $570 \mathrm{~nm}$ with a reference wavelength of $630 \mathrm{~nm}$.

Cell cycle analysis. The LX-2 cells were incubated for $24 \mathrm{~h}$ in a 5-ml cell culture flask in DMEM with $10 \%$ FBS medium and then cultured in serum-free medium for an additional $24 \mathrm{~h}$. The cells were treated with $40 \mathrm{mM}$ NTau for $48 \mathrm{~h}$ and then fixed with $70 \%$ ice-cold ethanol. The fixed cells were permeabilized with PBST (137 $\mathrm{mM} \mathrm{NaCl}, 3 \mathrm{mM} \mathrm{KCl}, 8 \mathrm{mM}$ $\mathrm{NA}_{2} \mathrm{HPO}_{4}$ and $0.1 \%$ Tween-20; $\mathrm{pH} 7.4$ ) and then stained with $100 \mathrm{mg} / \mathrm{l}$ RNase A and $50 \mathrm{mg} / \mathrm{l}$ propidium iodide (PI) in the dark for cell cycle analysis. Cell cycle analysis was performed on a Coulter ELITE flow cytometer (BD Biosciences, Franklin Lakes, NJ, USA) through a 488-nm (LP) filter. The data were analyzed using the MultiCycle AV software (Phoenix Flow Systems, San Diego, CA, USA) for cell cycle distribution.

Apoptosis detection. LX-2 cells were incubated for $48 \mathrm{~h}$ in the presence $40 \mathrm{mM}$ NTau and then re-suspended in $100 \mu \mathrm{l}$ buffer containing calcium ions. The cells were treated with $5 \mu \mathrm{l}$ Annexin V-FITC dye for 20-30 min and then with $5 \mu 1$ PI dye for $5 \mathrm{~min}$. The cell concentration was adjusted to $\sim 1 \mathrm{x} 10^{5} / \mathrm{ml}$ by adding the appropriate amount of calcium ion-containing buffer. The cells were loaded on a flow cytometer (Coulter ${ }^{\circledR}$ Elite) within $1 \mathrm{~h}$. Apoptotic analysis was performed using a Coulter ${ }^{\circledR}$ Elite flow cytometer (Beckman Coulter, Miami, FL,
USA) with excitation and emission wavelengths of 488 and $530 \mathrm{~nm}$, respectively.

Two-dimensional electrophoresis (2DE). LX-2 cell lysates were collected and centrifuged at $14,000 \mathrm{rpm}$ for $10 \mathrm{~min}$ at $4^{\circ} \mathrm{C}$. The supernatants were analyzed by $2 \mathrm{DE}$, and isoelectric focusing (IEF) was performed using an IPGphor IEF system (Bio-Rad, Hercules, CA, USA). The protein extract $(200 \mu \mathrm{g})$ was mixed with rehydration buffer to $350 \mu \mathrm{l}$ and loaded onto 17-cm, immobilized, nonlinear $\mathrm{pH}$ gradient (IPG) dry strips (pH 4-7; Bio-Rad). The IPG strips were equilibrated for $15 \mathrm{~min}$ in an equilibration buffer (6 M urea, 30\% glycerol, $2 \%$ SDS and $50 \mathrm{mM}$ Tris- $\mathrm{HCl}$ ) containing $10 \mathrm{mg} / \mathrm{ml}$ dithiothreitol (DTT), followed by $15 \mathrm{~min}$ in an equilibration buffer containing $40 \mathrm{mg} / \mathrm{ml}$ iodoacetamide. Following equilibration, strips were applied to $12 \%$ sodium dodecyl sulphate-polyacrylamide gel electrophoresis (SDS-PAGE) gels and sealed with agarose sealing solution. Following electrophoresis, the SDS-PAGE gels were silver stained. Stained gels were scanned using an image scanner (Amersham Biosciences, Piscataway, NJ, USA) in transmission mode. Analysis of the gels was accomplished using the PDQuest analysis software (Bio-Rad) including background subtraction, spots detection and the establishment of a reference gel. Protein spots were selected based on the criterion of $>2$-fold variation of expression between NTau-treated and control samples.

Mass spectrometry (MS) analysis. MS was performed on a UPLC-ESI-MS/MS (Waters/Micromass, Manchester, UK) equipped with an electrospray ionization (ESI) source. Data-dependent analysis was employed (the 3 most abundant ions in each cycle): $1 \mathrm{sec}$ MS m/z 350-1,600, and max $5 \mathrm{sec} \mathrm{MS} / \mathrm{MS} \mathrm{m} / \mathrm{z}$ 50-2,000 (continuum mode), with $50 \mathrm{sec}$ dynamic exclusion. The positive-ion mode was employed, and the capillary voltage was set at $3.0 \mathrm{kV}$. The cone voltage was set at $35 \mathrm{~V}$ to investigate the intensities and distribution of ions in the mass spectra of samples. The MS/MS spectra were processed, searched using ProteinLynx Global SERVER ${ }^{\text {TM }}$ (PLGS) v2.3 (Waters/Micromass), and searched against the NCBInr database by MASCOT (http://www.matrixscience. co.uk) using the following constraints: only tryptic peptides with up to 2 missed cleavage sites were allowed and 0.3-Da mass tolerances for MS and MS/MS fragment ions. The results were filtered by a peptide score of $\geq 30$.

Western blot analysis. Total proteins in LX-2 cell lysates were quantified by the Bradford method $(9,10)$ and analyzed on $12 \%$ SDS-PAGE gels. The gels were transferred onto a nitrocellulose membrane using a Trans-Blot SD apparatus (Bio-Rad). The membrane was incubated with anti-IgY [dilution 1:1,000 in Tris-buffered saline (TBS)] followed by incubation with secondary antibody (dilution 1:5,000 in TBS). Visualization of the protein bands was achieved by the chemiluminescence method, and the films were developed and fixed. Glyeraldehyde-3-phosphate dehydrogenase (GAPDH) was used as an internal reference.

Gene ontology (GO) analysis. GO is a stratified tree structure for the analysis of the functions of genes and proteins (11). The 3 hierarchical principles of GO are 'Biological Process' 
Table I. Primers used for real-time PCR of the $\operatorname{cox} 5 a, \operatorname{cox} 6 c, n d u f b 1, n d u f c 1$ and $\operatorname{tg} f \beta 1 / 1$ genes.

\begin{tabular}{|c|c|c|c|c|}
\hline Genes & Primer & Sequence $\left(5^{\prime}-3^{\prime}\right)$ & Temperature $\left({ }^{\circ} \mathrm{C}\right)$ & Product size (bp) \\
\hline \multirow[t]{2}{*}{$\operatorname{cox} 5 a$} & Forward & TAAACCGCATGGATGGGC & \multirow[t]{2}{*}{49} & \multirow[t]{2}{*}{177} \\
\hline & Reverse & AGTTCAAACTCATTTCCCTTTTATT & & \\
\hline \multirow[t]{2}{*}{$\operatorname{cox} 6 c$} & Forward & GGGGTTGCAGCTTTGTAT3 & \multirow[t]{2}{*}{49} & \multirow[t]{2}{*}{112} \\
\hline & Reverse & CAGCСТTCСТCATCTCCT & & \\
\hline \multirow[t]{2}{*}{$n d u f c l$} & Forward & CCGAATGCCAAACCTGAC & \multirow[t]{2}{*}{49} & \multirow[t]{2}{*}{127} \\
\hline & Reverse & ATTCCAGCCCATTTCTTC & & \\
\hline \multirow[t]{2}{*}{$n d u f b 1$} & Forward & TTCCCTGTTGCCCTTGGT & \multirow[t]{2}{*}{53.1} & \multirow[t]{2}{*}{158} \\
\hline & Reverse & AGCCGTTCATCACTCTTTCTGT & & \\
\hline \multirow[t]{2}{*}{$\operatorname{tg} \beta \beta 1 / 1$} & Forward & TTCTGCTGCGTCAGTTGC & \multirow[t]{2}{*}{57.4} & \multirow[t]{2}{*}{154} \\
\hline & Reverse & TGAGCGCCGAGATGTAGTT & & \\
\hline \multirow[t]{2}{*}{ gapdh } & Forward & GACCTGACCTGCCGTCTA & \multirow[t]{2}{*}{56} & \multirow[t]{2}{*}{148} \\
\hline & Reverse & AGGAGTGGGTGTCGCTGT & & \\
\hline
\end{tabular}

(BP), 'Cellular Component' (CC) and 'Molecular Function' (MF). We analyzed the functional distribution of differential gene expression and protein production over the 3 principles. To accurately detect significantly over-represented GO terms, the Database for Annotation, Visualization, and Integrated Discovery (DAVID) tool (http://david.abcc.ncifcrf.gov) was used by analyzing into the fourth layers (12). The threshold value of group membership counts was set at 3 , and the EASE score was set at 0.1 . Then, the functional annotation clustering tool in DAVID was used to cluster functionally related annotations into groups for a 2D view of the related gene-term relationship (12). We ranked the importance of annotation groups with an enrichment score. In addition, we also used the DAVID tool to map differential gene expression and protein production into the Kyoto Encyclopedia of Genes and Genomes (KEGG) pathways database (13) to facilitate the biological interpretation in a network context. Fisher's exact test was used and $\mathrm{P}<0.05$ was considered to indicate a statistically significant difference.

Microarray analysis. A genome-wide 70-mer oligonucleotide microarray including 22,000 well-characterized human genes (14) was obtained from CapitalBio Corporation (Beijing, China) to investigate the expression profiling of NTau-treated LX-2 cells. The cDNA targets were prepared from $5 \mu \mathrm{g}$ total RNA and were labeled with fluorescent dyes (Cy5 and Cy3-dCTP) by the Eberwine linear RNA amplification method and a subsequent enzymatic reaction (14). The RNA samples from the NTau-treated LX-2 cells were labeled with Cy3-dUTP and named as ' 1 .' The RNA samples from the blank control cells were labeled with Cy5-dUTP and named as '2.' We then prepared the hybridization solution in hybridization buffer (25\% formamide, 3X SSC, $0.2 \%$ SDS and 5X Denhardt's solution) and hybridized it with sample at $42^{\circ} \mathrm{C}$ overnight in a humid environment. The hybridized microarrays were scanned with a confocal LuxScan ${ }^{\mathrm{TM}}$ scanner (CapitalBio Corporation, Beijing, China) at 2 wavelengths to detect emission from both Cy3 and Cy5. The images obtained were then analyzed using LuxScan $^{\mathrm{TM}} 3.0$ software (CapitalBio Corporation). Then, an intensity-dependent locally weighted scatterplot-smoothing regression (LOWESS) algorithm was used to normalize the 2-channel ratio values by an $\mathrm{R}$ package (15).
Gene set enrichment analysis (GSEA). GSEA is a software (https://www.broad.harvard.edu/gsea/) for searching in predefined gene sets (ex. pathways) and ranking genes to identify significant biological changes in microarray data sets (16). First, ratios of filtered genes were operated by logarithm function to the base 1.5, and the input 'rnk' file was made based on the ranked value of $\log _{1.5}$ (ratio). These expression matrices were exported to GSEA software and searched against the background dataset of 'c4: computational gene sets' with 'GSEA Pre-ranked' option. All the default settings except 'gene set permutation' with 1,000 iterations were used for the analysis.

RNA extraction and real-time polymerase chain reaction (PCR). Total RNA was extracted using TRIzol (Invitrogen, Gaithersburg, MD, USA) reagent according to the manufacturer's instructions. RNA was purified using the NucleoSpin RNA Clean-up kit (Macherey-Nagel, Düren, Germany). RNA quality from each sample was assessed by visualization of the $28 \mathrm{~S} / 18 \mathrm{~S}$ ribosomal RNA ratio on $1 \%$ agarose gels. First-strand cDNA was synthesized using $1 \mu \mathrm{g}$ total RNA in a $20-\mu 1$ final volume by reverse transcription utilizing ReverTra Ace ${ }^{\circledR}$ reverse transcriptase (Toyobo, Co., Ltd., Osaka, Japan) with random hexamer primers or oligo $(\mathrm{dT})_{18}$ primers (Invitrogen). PCR was performed using $0.5 \mu \mathrm{l}$ cDNA, with specific primers and Ex Taq ${ }^{\mathrm{TM}}$ Polymerase (Takara Bio, Inc., Otsu, Japan) in a volume of $12.5 \mu \mathrm{l}$. The PCR products were then separated on $1.5 \%$ agarose gels. The real-time PCR reactions were performed using $\mathrm{iQ}^{\mathrm{TM}} \mathrm{SYBR}^{\circledR}$-Green Supermix kit according to the manufacturer's instructions (Bio-Rad). RNA was amplified using the ABI Prism 7500 Sequence Detection system (Applied Biosystems, Carlsbad, CA, USA). The primers (Invitrogen) are shown in Table I. For all the real-time PCR experiments, negative controls were a non-reverse transcriptase reaction, and a non-sample reaction (data not shown). GAPDH was amplified as an internal standard.

Statistical analysis. All experiments were performed $\geq 3$ times with triplicate measurements, and data are expressed as the mean \pm standard error of the mean (SEM). Statistical analysis was performed using the R software (version 2.9.2, http:// www.r-project.org/). Student's t-test was performed to evaluate 


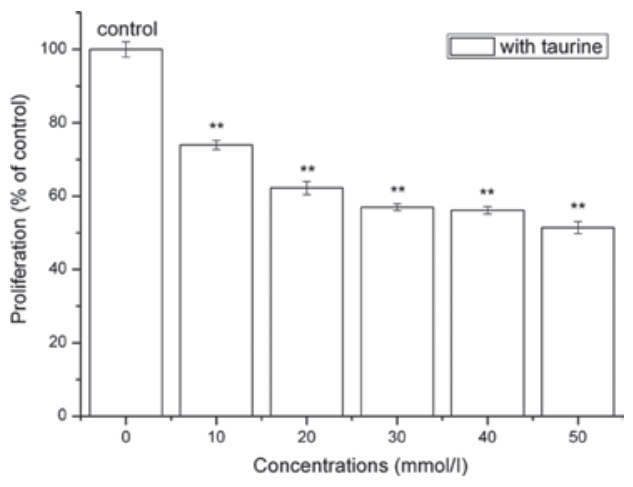

Figure 1. Cell proliferation analysis by MTT assay. Different concentrations of taurine $(0,10,20,30,40$ and $50 \mathrm{mmol} / \mathrm{l})$ were used to treat hepatic stellate cells (HSCs). The experiments were repeated 5 times for each concentration and the proliferation rates are presented as the means \pm standard deviation (SD); ${ }^{* *} \mathrm{P}<0.005$, vs. the blank control (one-way ANOVA).

the differences of cell proliferation rate among the groups treated with different concentrations of NTau. The Student's t-test was also used to evaluate the gene expression changes in real-time PCR results between 2 phenotypes. The Chi-square test was used to assess the effect of NTau on the cell cycle of HSCs. Fisher's exact test was used to identify the significant GO terms of target genes relative to genome backgrounds. $\mathrm{P}<0.05$ was considered to indicate a statistically significant difference.

\section{Results}

Effect of NTau on cell proliferation and cell cycle of HSCs. As shown in Fig. 1, an inverse correlation between the proliferation of LX-2 cells and the concentration of NTau was detected. Treatment with different concentrations of NTau significantly inhibited the growth of LX-2 cells when compared with that of the non-treated control cells (one-way ANOVA, $\mathrm{P}<0.005$ ). The growth rate of LX-2 cells in the presence of $30-40 \mathrm{mmol} / 1 \mathrm{NTau}$ was reduced by $43 \%$ compared with that of the non-treated cells. As assessed by cell cycle analysis, the percentage of LX-2 cells in the G0/G1 phase increased from 43.9 to $50.9 \%$ in response to $40 \mathrm{mmol} / 1 \mathrm{NTau}$, while exposure of the LX-2 cells to $40 \mathrm{mmol} / \mathrm{l} \mathrm{NTau}$ for $48 \mathrm{~h}$ caused a 1 - to 3 -fold reduction $(\mathrm{P}<0.04)$ of the $\mathrm{S}-$ and $\mathrm{G} 2 / \mathrm{M}$-phase cell populations compared with those in the non-treated control cells.

Detection of NTau-induced apoptosis in HSCs. To investigate the mechanism underlying the growth-inhibitory effects of NTau on HSCs, the cell-apoptosis analysis of NTau-treated LX-2 cells was performed. As shown in Fig. 2, the increase in apoptotic cells in the NTau-treated LX-2 cells was comparable to that in the non-treated control cells $(13.6 \pm 3.3$ vs. $4.65 \pm 1.1 \%, \mathrm{P}<0.05)$. These findings suggest that the induction of cellular apoptosis contributed, at least in part, to the HSC growth-inhibiting effects of NTau.

Identification of differentially expressed proteins in HSCs following NTau treatment. The protein profiles of HSCs were analyzed by $2 \mathrm{DE}$ and visualized using the PDQuest image analysis software. By comparing the protein profiles of the NTau-treated and non-treated control HSCs, 15 differentially expressed proteins were successfully identified. We picked up protein spots in the $2 \mathrm{D}$ gel image with identified changes and prepared them for MS analysis. Table II summarizes the code name, relative molecular weight, isoelectric point, and peptide fragment coverage for the differentially expressed proteins. The most significantly upregulated expression was of CAA32649, MYL9, PSMB6, ANXA1, MDH1, HSPB1, LASP1, LOC100134370, and SOD1, while ATP5H, BAF82933, ECHS1, PRDX2, HNRNPA2B1, and BAG36698 showed the most markedly downregulated protein expression.

Functional analysis of differentially expressed proteins in HSCs following NTau treatment and validation of proteomic data. We analyzed the functional enrichment of the differentially expressed proteins using the DAVID tool based on their annotation keywords from the UniProt database (17). Significant protein functions terms and corresponding proteins were identified (Fisher's exact test, $\mathrm{P}<0.05$ ). As shown in Table III, proteins that corresponded to the category of 'acetylation,' 'direct protein sequencing,' 'antioxidant,' 'cytoplasm' and 'oxidoreductase' were significantly affected in the NTau-treated HSCs.

Among the differentially expressed proteins identified successfully by MS, the upregulated proteins ANXA1 and PSMB6 and the downregulated proteins ECHS1 and PRDX2 were selected and subjected to western blot analysis. As shown in Fig. 3, NTau treatment significantly upregulated the expression of ANXA1 and downregulated the expression of ECHS1 and PRDX2 in HSCs. The expression of PSMB6 was not significantly different following NTau treatment. Therefore, western blot analysis of the differentially expressed proteins confirmed the reliability and validity of the proteomic high throughput experiments.

Differential gene expression in HSCs following NTau treatment. According to the filtering principles described above, 6,109 normally expressed genes (28.38\%) with high confidence were screened among 21,522 genes. By applying the threshold of 1.5-fold change for an intensity ratio of NTau-treated vs. control HSCs, 658 genes (3.06\%) were shown to be differentially expressed in NTau-treated HSCs. Among the differentially expressed genes, 241 were upregulated (1.12\%) and 417 were 


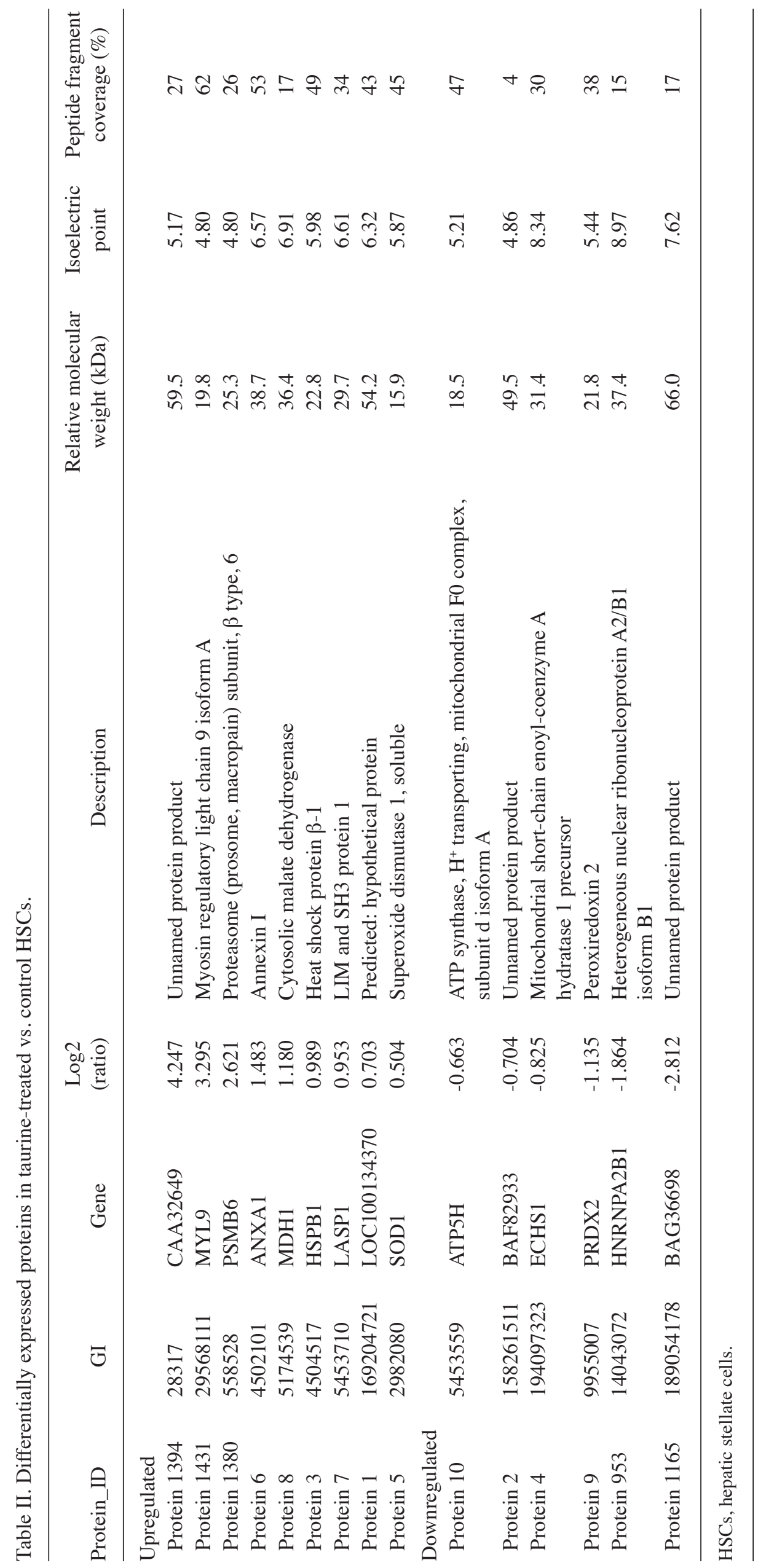


Table III. Functional enrichment analysis based on the annotation keywords of proteins.

\begin{tabular}{|c|c|c|c|}
\hline Term & P-value & Proteins & Fold enrichment \\
\hline Acetylation & $6.8 \mathrm{E}-09$ & $\begin{array}{l}\text { PRDX2, ATP5H, MDH1, MYL9, } \\
\text { HSPB1, LASP1, SOD1, ECHS1 }\end{array}$ & 20.79 \\
\hline Direct protein sequencing & $1.0 \mathrm{E}-06$ & $\begin{array}{l}\text { PRDX2, PSMB6, ATP5H, MDH1, HSPB1, } \\
\text { HNRNPA2B1, SOD1, ECHS1 }\end{array}$ & 10.04 \\
\hline Antioxidant & $5.5 \mathrm{E}-03$ & PRDX2, SOD1 & $328.10 .$. \\
\hline Cytoplasm & $5.9 \mathrm{E}-03$ & $\begin{array}{l}\text { PRDX2, PSMB6, MDH1, LASP1, } \\
\text { SOD1, ANXA1 }\end{array}$ & 4.08 \\
\hline Oxidoreductase & 4.7E-02 & PRDX2, MDH1, SOD1 & 7.70 \\
\hline
\end{tabular}

Fisher's exact test, $\mathrm{P}<0.05$

Table IV. Top 9 upregulated and top 10 downregulated genes in taurine-treated vs. control HSCs.

\begin{tabular}{|c|c|c|c|}
\hline Gene & RefSeq_ID & Description & $\log _{1.5}($ ratio $)$ \\
\hline \multicolumn{4}{|c|}{ Top 9 upregulated } \\
\hline hdac3 & NM_003883 & Histone deacetylase 3 & 1.8950 \\
\hline $\operatorname{tg} f \beta 1 i 1$ & NM_015927 & Transforming growth factor $\beta 1$-induced transcript 1 & 1.8773 \\
\hline tmem120a & NM_031925 & $\begin{array}{l}\text { Transmembrane protein induced } \\
\text { by tumor necrosis factor- } \alpha\end{array}$ & 1.8526 \\
\hline cyp $2 e 1$ & NM_000773 & $\begin{array}{l}\text { Cytochrome P450, family } 2 \text {, } \\
\text { subfamily E, polypeptide } 1\end{array}$ & 1.7893 \\
\hline$h r h 1$ & NM_000861 & Histamine receptor $\mathrm{H} 1$ & 1.7789 \\
\hline selt & NM_016275 & Selenoprotein T & 1.6962 \\
\hline$c c d c 86$ & NM_024098 & Coiled-coil domain containing 86 & 1.6648 \\
\hline mif4gd & NM_020679 & MIF4G domain containing & 1.6570 \\
\hline psap & NM_002778 & $\begin{array}{l}\text { Prosaposin (variant Gaucher disease and variant } \\
\text { metachromatic leukodystrophy) }\end{array}$ & 1.6430 \\
\hline \multicolumn{4}{|c|}{ Top 10 downregulated } \\
\hline nucb2 & NM_005013 & Nucleobindin 2 & -2.7135 \\
\hline adam 9 & NM_003816 & $\begin{array}{l}\text { A disintegrin and metalloproteinase } \\
\text { domain } 9(\text { meltrin } \gamma)\end{array}$ & -2.7336 \\
\hline tpp2 & NM_003291 & Tripeptidyl peptidase II & -2.7350 \\
\hline abcel & NM_002940 & $\begin{array}{l}\text { ATP-binding cassette, sub-family E (OABP), } \\
\text { member } 1\end{array}$ & -2.7956 \\
\hline mtif2 & NM_002453 & Mitochondrial translational initiation factor 2 & -2.8257 \\
\hline pkn2 & NM_006256 & Protein kinase N2 & -3.2762 \\
\hline$n d u f b 1$ & NM_004545 & $\begin{array}{l}\text { NADH dehydrogenase (ubiquinone) } \\
1 \beta \text { subcomplex, } 1,7 \mathrm{kDa}\end{array}$ & -3.6023 \\
\hline brcc3 & NM_024332 & BRCA1/BRCA2-containing complex, subunit 3 & -3.6603 \\
\hline adamtsl3 & NM_207517 & ADAMTS-like 3 & -3.7298 \\
\hline adam9 & NM_003816 & $\begin{array}{l}\text { A disintegrin and metalloproteinase } \\
\text { domain } 9(\text { meltrin } \gamma)\end{array}$ & -5.9415 \\
\hline
\end{tabular}

HSCs, hepatic stellate cells.

downregulated (1.94\%). The top 9 upregulated and the top 10 downregulated genes are presented in Table IV.

Functional categorization and clustering for the differentially expressed genes. The differentially expressed genes were then categorized according to their GO function using the DAVID tool. Six function categories of 'Molecular Function' (MF), 51 function categories of 'Cellular Component' (CC), and 42 function categories of 'Biological Process' (BP) were significantly enriched in the differentially expressed genes 
Table V. Functional annotation clustering analysis of the differentially expressed genes.

\begin{tabular}{l} 
Category \\
\hline Functional group 1 \\
GOTERM_CC_4 \\
GOTERM_CC_4 \\
GOTERM_CC_4 \\
GOTERM_CC_4 \\
Functional group 2 \\
GOTERM_CC_4 \\
GOTERM_CC_4 \\
GOTERM_CC_4 \\
GOTERM_CC_4 \\
GOTERM_CC_4 \\
GOTERM_CC_4 \\
GOTERM_CC_4 \\
GOTERM_CC_4 \\
GOTERM_CC_4 \\
GOTERM_CC_4
\end{tabular}

Functional group 3 GOTERM_CC_4 GOTERM_CC_4 GOTERM_CC_4 GOTERM_CC_4 GOTERM_CC_4 GOTERM_CC_4 GOTERM_MF_4 GOTERM_MF_4

\section{GOTERM_BP_4}

GOTERM_BP_4

Functional group 4 GOTERM_CC_4 GOTERM_CC_4 GOTERM_CC_4 GOTERM_CC_4

Functional group 5 GOTERM_BP_4 GOTERM_BP_4 GOTERM_BP_4 GOTERM_BP_4 GOTERM_BP_4

Functional group 6 GOTERM_BP_4 GOTERM_BP_4 GOTERM_MF_4 GOTERM_BP_4 GOTERM_BP_4 GOTERM_BP_4 GOTERM_BP_4 GOTERM_BP_4
Term

Percentage

P-value

Fold enrichment

Geo: 4.6040E-15

GO:0044424-intracellular part

GO:0043229-intracellular organelle

GO:0043231-intracellular membrane-bound organelle

GO:0005634-nucleus

$\begin{array}{lll}0.6873 & 4.45 \mathrm{E}-22 & 1.2591 \\ 0.5937 & 7.00 \mathrm{E}-17 & 1.2999 \\ 0.5349 & 5.09 \mathrm{E}-16 & 1.3499 \\ 0.3254 & 2.83 \mathrm{E}-05 & 1.2661 \\ & & \\ 0.0603 & 2.33 \mathrm{E}-11 & 3.6125 \\ 0.0825 & 2.28 \mathrm{E}-10 & 2.6796 \\ 0.0778 & 6.55 \mathrm{E}-10 & 2.6988 \\ 0.0540 & 9.73 \mathrm{E}-10 & 3.4607 \\ 0.1127 & 2.203 \mathrm{E}-09 & 2.1238 \\ 0.0333 & 2.326 \mathrm{E}-09 & 5.26001 \\ 0.0603 & 4.029 \mathrm{E}-09 & 3.0154 \\ 0.0619 & 4.501 \mathrm{E}-09 & 2.9486 \\ 0.1429 & 5.406 \mathrm{E}-09 & 1.8718 \\ 0.0254 & 2.795 \mathrm{E}-08 & 6.2282\end{array}$

Geo: 1.4710E-9

GO:0019866-organelle inner membrane

GO:0031967-organelle envelope

GO:0044429-mitochondrial part

GO:0005743-mitochondrial inner membrane

GO:0005739-mitochondrion

GO:0044455-mitochondrial membrane part

GO:0031966-mitochondrial membrane

GO:0005740-mitochondrial envelope

GO:0031090-organelle membrane

GO:0005746-mitochondrial respiratory chain

Geo: $5.5926 \mathrm{E}-7$

GO:0005743-mitochondrial inner membrane

GO:0044455-mitochondrial membrane part

GO:0005746-mitochondrial respiratory chain

GO:0030964-NADH dehydrogenase complex (quinone)

GO:0005747-mitochondrial respiratory chain complex I

GO:0045271-respiratory chain complex I

GO:0003954-NADH dehydrogenase activity

GO:0016655-oxidoreductase activity,

acting on NADH or NADPH, quinone or

similar compound as acceptor

GO:0006120-mitochondrial electron transport,

NADH to ubiquinone

GO:0042773-ATP synthesis coupled electron transport

0.0540

0.0333

0.0254

0.0175

0.0175

0.0175

0.0190

0.0190

9.73E-10

2.33E-09

2.80E-08

$1.56 \mathrm{E}-06$

$1.56 \mathrm{E}-06$

$1.56 \mathrm{E}-06$

$1.56 \mathrm{E}-06$

$4.22 \mathrm{E}-06$

0.0159

2.38E-05

6.2590

0.0175

8.01E-05

4.8297

Geo: 3.9490E-6

GO:0044428-nuclear part

GO:0044451-nucleoplasm part

GO:0005654-nucleoplasm

GO:0031981-nuclear lumen

Geo: $1.9522 \mathrm{E}-4$

GO:0046907-intracellular transport

GO:0015031-protein transport

GO:0045184-establishment of protein localization

GO:0006886-intracellular protein transport

GO:0051649-establishment of cellular localization

Geo: 0.0010

GO:0051246-regulation of protein metabolic process

GO:0009889-regulation of biosynthetic process

GO:0003743-translation initiation factor activity

GO:0006446-regulation of translational initiation

GO:0006417-regulation of translation

0.1175

$1.01 \mathrm{E}-08$

2.0090

0.0540

$1.50 \mathrm{E}-05$

2.2883

0.0587

$2.47 \mathrm{E}-05$

2.1445

0.0698

6.50E-05

1.9002

0.0714

0.0698

9.11E-05

1.8540

0.0714

0.0001

1.8571

1.7769

0.0460

0.0002
0.0003

2.1064

0.0794

0.0004

1.6695

$\begin{array}{ll}0.0381 & 0.0003 \\ 0.0286 & 0.0007 \\ 0.0159 & 0.0007 \\ 0.0127 & 0.0008 \\ 0.0254 & 0.0009 \\ 0.0190 & 0.0019 \\ 0.0254 & 0.0020 \\ 0.0571 & 0.0028\end{array}$

2.2997

2.5580

4.0893

5.1161

2.7050

3.0432

2.4904

1.6837 
Table V. Continued.

\begin{tabular}{rlccc}
\hline Category & \multicolumn{1}{c}{ Term } & Percentage & P-value & Fold enrichment \\
\hline Functional group 7 & Geo: 0.0073 & & & \\
GOTERM_CC_4 & GO:0012505-endomembrane system & 0.0825 & 0.0015 & 1.5603 \\
GOTERM_CC_4 & GO:0005783-endoplasmic reticulum & 0.0730 & 0.0018 & 1.6022 \\
GOTERM_CC_4 & GO:0044432-endoplasmic reticulum part & 0.0444 & 0.0131 & 1.6327 \\
GOTERM_CC_4 & GO:0042175-nuclear envelope-endoplasmic & 0.0397 & 0.0208 & 1.6219 \\
& reticulum network & & & \\
GOTERM_CC_4 & GO:0005789-endoplasmic reticulum membrane & 0.0381 & 0.0291 & 1.5893 \\
Functional group8 & Geo: 0.0225 & & & \\
GOTERM_BP_4 & GO:0007067-mitosis & 0.0254 & 0.0106 & 2.0735 \\
GOTERM_BP_4 & GO:0000087-M phase of mitotic cell cycle & 0.0254 & 0.0115 & 2.0554 \\
GOTERM_BP_4 & GO:0000279-M phase & 0.0270 & 0.0359 & 1.7425 \\
GOTERM_BP_4 & GO:0022403-cell cycle phase & 0.0302 & 0.0588 & 1.5700 \\
Functional group 9 & Geo: 0.0346 & & & \\
GOTERM_CC_4 & GO:0005635-nuclear envelope & 0.0206 & 0.0247 & 2.0575 \\
GOTERM_CC_4 & GO:0005637-nuclear inner membrane & 0.0063 & 0.0265 & 6.0643 \\
GOTERM_CC_4 & GO:0044453-nuclear membrane part & 0.0127 & 0.0467 & 2.4257 \\
GOTERM_CC_4 & GO:0031965-nuclear membrane & 0.0159 & 0.0469 & 2.1180 \\
\hline
\end{tabular}

GO, gene ontology; CC, cellular component; MF, molecular function; BP, biological process.

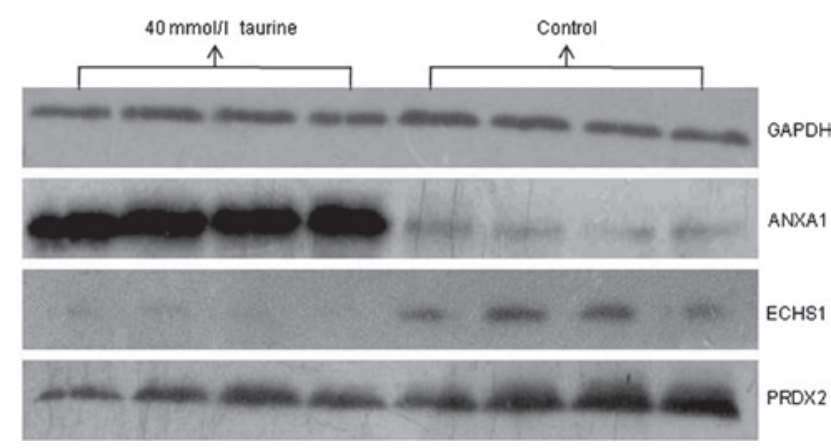

Figure 3. Results of validation by western blot analysis for the differentially expressed proteins. GAPDH was used as an internal reference. The experiment was repeated 4 times for each protein. The four columns on the right side represent the control group, and the four columns on the left side represent the taurine-treated group.

(Fisher's exact test, $\mathrm{P}<0.05)$. Fig. 4 lists the top 5 significantly enriched GO terms identified after screening with a threshold of false discovery rate (FDR) of $<0.01$. Functional categorization of the GO terms demonstrated that these differentially expressed genes were strongly associated with biological processes of 'NADH reduction and oxidation reaction' and 'RNA processing'. Based on the analysis above, the heuristic fuzzy clustering was used to classify the groups of similar annotations by the $\kappa$ statistic values (18). Table $\mathrm{V}$ provides a visualized network of the 9 significantly enriched GO terms (geometric mean of member's $\mathrm{P}$-value, $\mathrm{P}<0.05$ ). Clusters 1,2 , $3,4,7$ and 9 correspond to the categories of 'cellular transport and translation,' 'oxidant reaction' and 'mitosis process,' respectively. This suggests a crucial role of these biological processes in NTau-treated HSCs.
Pathway-based (GSEA) microarray analysis of NTau-treated HSCs and validation of microarray analysis. mRNA expression profiling using GSEA microarrays and quantitative PCR (qPCR) was performed in NTau-treated HSCs. Analysis of the expression of individual mRNAs demonstrated 2 different patterns of expression. A number of genes including $n u c b 2$, adam9, tpp2, mtif2, abce1, pkn2, ndufb1, brcc3, adamtsl3 and $g b p l$ correlated with reduction and oxidation of NADH showed a reduced expression in the NTau-treated HSCs. The second group, consisting of genes related to cell proliferation and cell cycle regulation including hdac3, tgf $\beta 1$ il and hrhl, showed an increased expression in NTau-treated HSCs. We selected 2 of the gene sets 'Module 62' and 'MORF_RAD21' to illustrate the enrichment scores as shown in Fig. 5, which was driven by the group of genes within a gene set that showed the highest correlation with NTau treatment.

Five selected candidate genes, snrpe, hnrph3, eiflay, nucb2 and vim, were used as reference genes were assayed by qPCR in order to confirm the expression profiles found using microarray analysis (Fig. 6). Another 5 selected genes, cox $5 a$, cox6c, $n d u f b l, n d u f c l$ and $t g f \beta 1 i l$, were assayed to identify the difference between NTau-treated and control HSCs. The primers designed for real-time PCR are listed in Table I. As shown in Fig. 7, tgf $\beta 1 i 1$ mRNA showed a significantly increased expression level $(2.26 \pm 0.41$ vs. $1, P=0.01)$, which coincided with pathway-based (GSEA) microarray analysis progression in the regulation of cell proliferation group. However, cox $5 a$ mRNA $\left(0.62 \pm 0.03\right.$ vs. $\left.1, \mathrm{P}=2 \times 10^{-4}\right), \operatorname{cox} 6 c \mathrm{mRNA}(0.51 \pm 0.07$ vs. 1 , $\left.\mathrm{P}=1.3 \times 10^{-3}\right)$, ndufbl mRNA( $0.53 \pm 0.07$ vs. $\left.1, \mathrm{P}=2.7 \times 10^{-5}\right)$, and $n d u f c l$ mRNA $\left(0.45 \pm 0.06\right.$ vs. $\left.1, \mathrm{P}=1.1 \times 10^{-3}\right)$ showed a reduced expression in the NTau-treated HSCs, which are known to play a role in the regulation of NADH dehydrogenase (ubiquinone) activity, which is involved in NADH oxidation. 


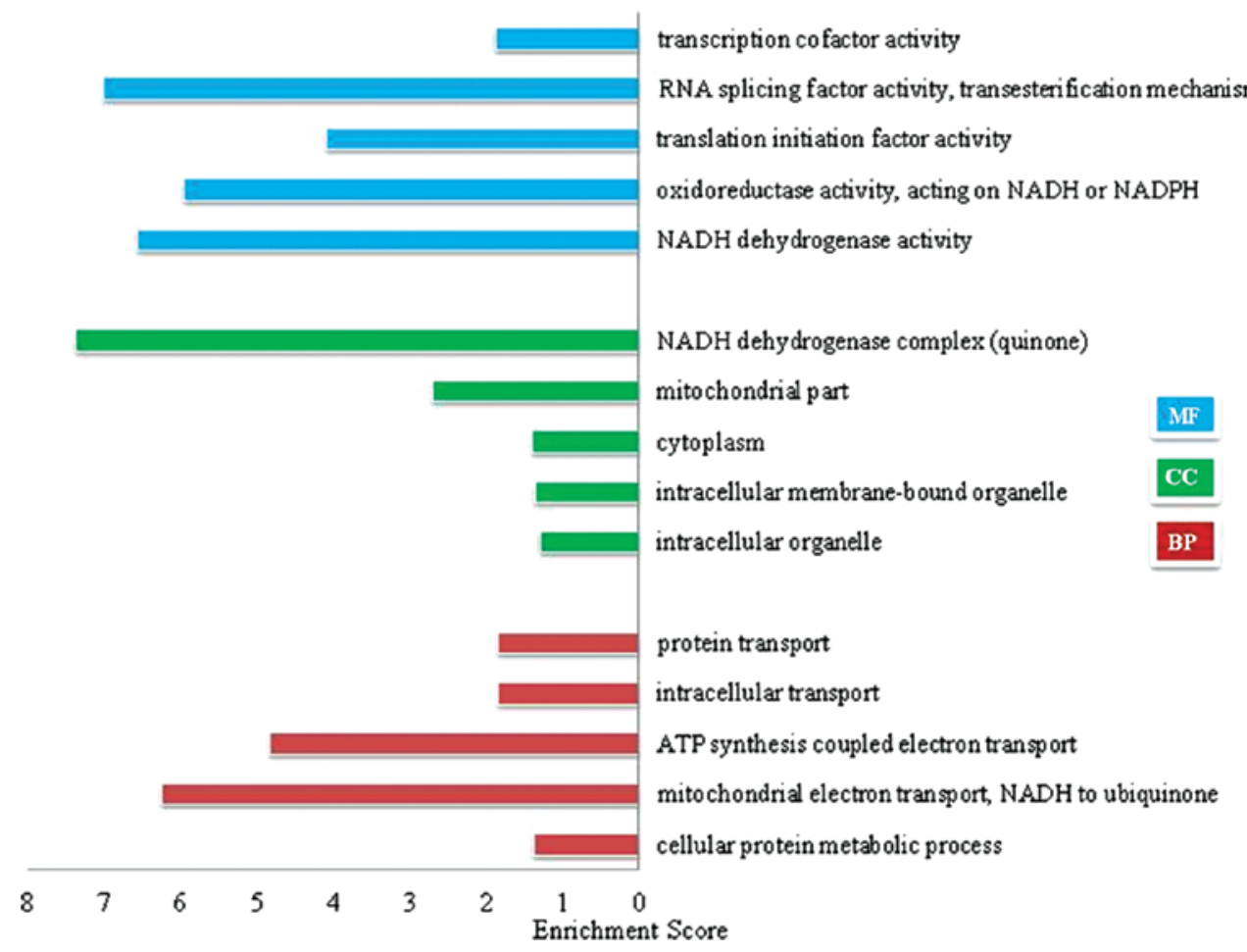

Figure 4. Functional category enrichment analysis of the differentially expressed genes. The y-axis shows significantly enriched gene ontology (GO) terms relative to the genome, and the x-axis shows the enrichment scores of these terms. Blue bars, 'Molecular Function' (MF) categories in GO; green bars, 'Cellular Component' (CO) categories in GO; red bars, 'Biological Process' (BP) categories in GO.
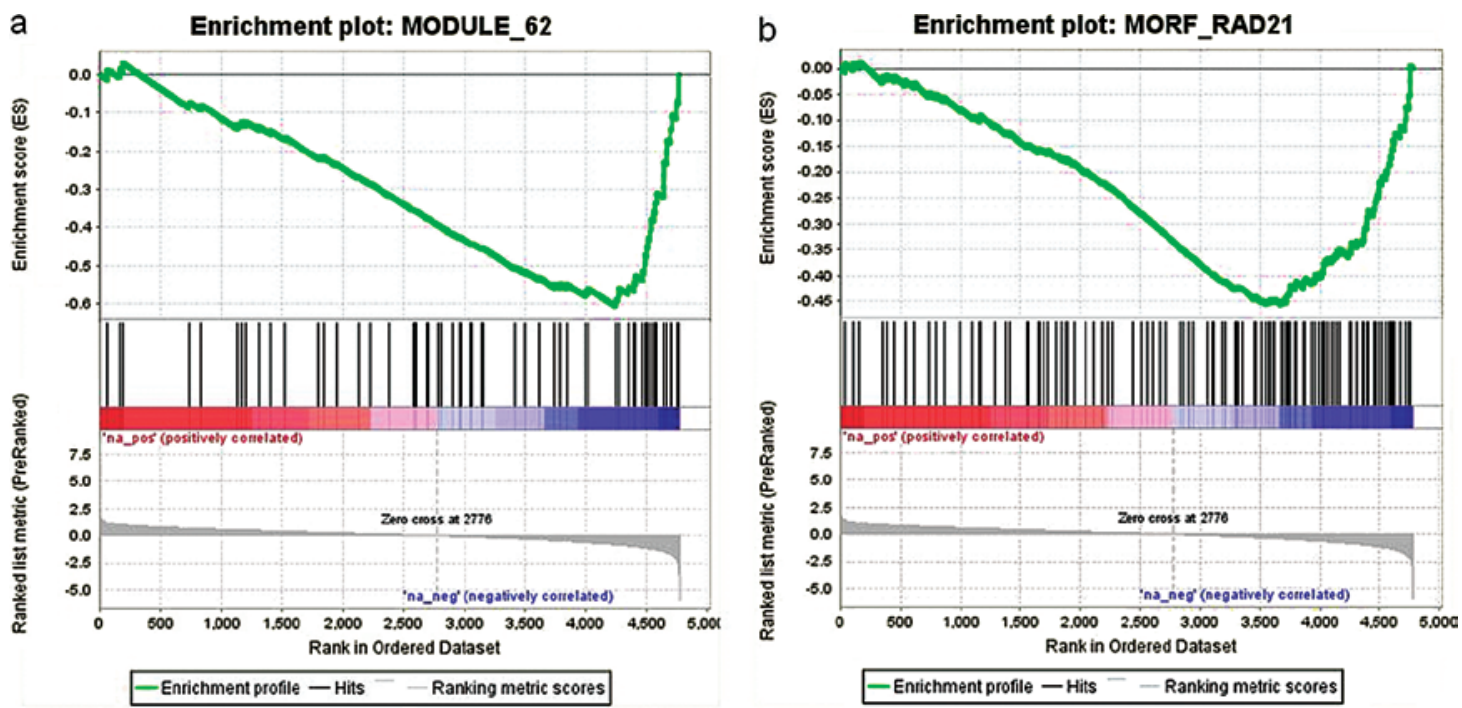

Figure 5. Gene set enrichment analysis (GSEA) score curves. Example of enrichment plots are shown for categories identified using GSEA as significantly enriched in negatively correlated genes in taurine-treated hepatic stellate cells (HSCs). Black bars represent the position of members of the category in the ranked list together with the running enrichment score (plotted in green). The leading edge is defined as those genes in the gene set that appear in the ranked list at or before the point where the enrichment score reaches its maximum deviation from 0 and may be interpreted as the core of a gene set that accounts for the enrichment signal. Examples shown are (a) Module 62, q=0.000 and (b) MORF_RAD21, $q<0.007$.

\section{Discussion}

Taurine is a sulfur-containing $\beta$-amino acid with several potential therapeutic applications 19,20$)$. Substantial progress has been made over the last 10-20 years in elucidating the bio-physiological function of taurine in the treatment of liver fibrosis. It has been reported that taurine is able to protect hepatocytes from chemically induced injury and mitigate the fibrosis of the liver $(6,7,21)$. Although recent findings suggest that taurine is able to inhibit the proliferation of HSCs and cause a G0/G1 phase arrest (2), the exact mechanism has not yet been fully elucidated. During chronic liver injury, HSCs undergo a phenotypic transformation with the acquisition of myofibroblast-like features by increased proliferation and synthesis of ECM components and play a pivotal role in the formation of fibrosis (22). Therefore, the inhibition of 


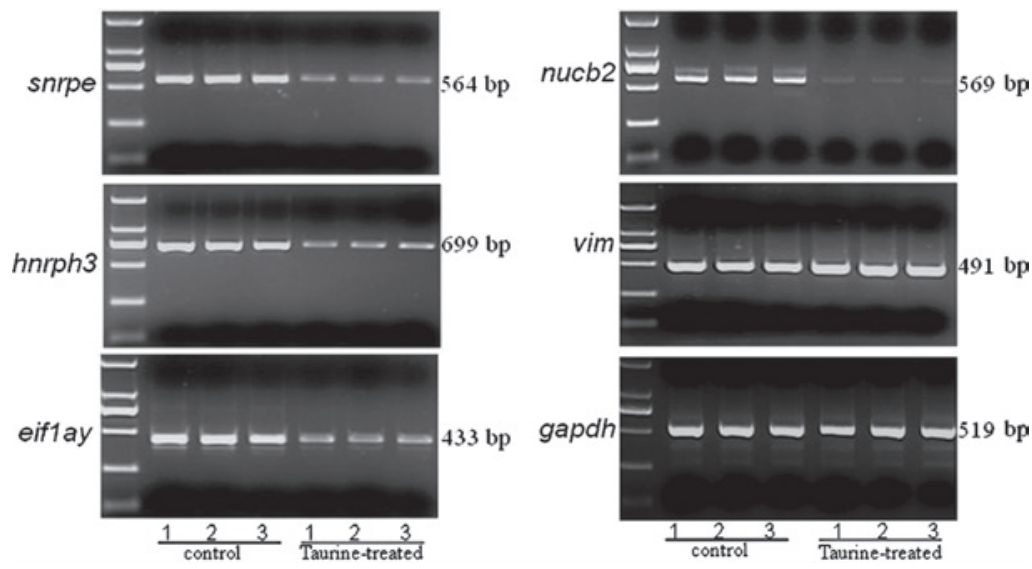

Figure 6. Reverse-transcription PCR validation. Microarray analysis demonstrated a decreased expression of the genes snrpe, hnrph3, eiflay and nucb2, and an increased expression of the gene vim. For each gene, PCR was performed on cDNA from taurine-treated hepatic stellate cells (HSCs) using primers to amplify the genes. The gapdh gene was used as a control. Numbers at the bottom of the figure indicate the repeats of experiments.

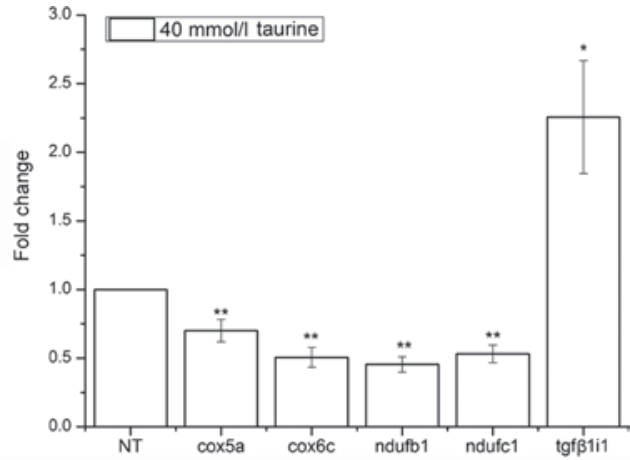

Figure 7. Quantitative determination of fold change of transcript levels by real-time PCR. Changes in transcript levels in taurine-treated hepatic stellate cells (HSCs) were confirmed by 4 -fold real-time PCR. The gapdh gene was used as reference. NT, normal HSCs. Five differentially expressed genes identified by microarray analysis, $\operatorname{cox} 5 a, \operatorname{cox} 6 c, n d u f b 1, n d u f c 1$ and $t g f \beta 1 i 1$ were selected to validate changes of the gene expression. Of these, $\operatorname{tg} f \beta 1 i 1$ was significantly upregulated in the taurine-treated compared with the control group $\left({ }^{*} \mathrm{P}<0.05\right)$. The remaining genes were significantly downregulated in the taurine-treated compared with the control group $\left({ }^{* *} \mathrm{P}<0.01\right)$.

HSC proliferation, the regulation of the cell cycle of HSCs, and the facilitation of HSC apoptosis are important therapeutic approaches for hepatic fibrosis-related liver diseases. The present study is congruent with the study by Chen (21), according to which taurine not only inhibits the proliferation of HSCs, but it is also able to promote HSC apoptosis.

The process of apoptosis is controlled by a diverse range of cell signals, which may originate either extracellularly or intracellularly. A cell initiates intracellular apoptotic signaling in response to stress, which may cause cell suicide. The binding of nuclear receptors by glucocorticoids, heat, radiation increase and intracellular calcium concentration, for example, by causing damage to the membrane, are all able to trigger the release of intracellular apoptotic signals by a damaged cell $(23,24)$.

The proteins ANXA1, ECHS1 and PRDX2 were selected for validation by western blot analysis. Functional analysis showed that these proteins were related to the biological processes of 'cellular apoptosis' and 'oxidation reaction.' Since flow cytometric analysis has shown that taurine-treated HSCs had a significantly increased apoptosis rate compared to the control group, the proteomic analysis may reveal the relevant mechanism. Upregulated protein Annexin I belongs to a family of $\mathrm{Ca}^{2+}$-dependent phospholipid-binding proteins, which are able to change the intracellular calcium concentration and cause apoptosis. Reactive oxygen species (ROS) are closely associated with apoptotic induction (25) and downregulate PRDX2 protein, a kind of antioxidant enzyme. Since altered cellular oxidation-reduction one of the key events in apoptosis that affects the mitochondria (26), it may be involved in oxidation reaction and HSC apoptosis.

GO analysis indicated that reduction and oxidation of $\mathrm{NADH}$ had significant functional enrichment. Fuzzy heuristic clustering of GO categories suggested that intracellular components of HSCs were influenced by NTau treatment. Furthermore, many differentially expressed genes were classified into function clusters relating to reduction-oxidation of mitochondrial NADH and mitosis. A variety of key events in apoptosis focus on the mitochondria, including the release of caspase activators, such as cytochrome $c$, changes in electron transport and altered cellular oxidation-reduction $(26,27)$.

Among the validated genes, upregulated $\operatorname{tg} f \beta 1 i 1$, which is transforming growth factor $\beta 1$-induced transcript 1 , is involved in the negative regulation of cell proliferation (28). This means that the higher its expression level, the slower the cell proliferation. Its upregulated level may indicate taurine's function in inhibiting the proliferation of LX-2 cells. The downregulated genes $\operatorname{cox} 6 c, \operatorname{cox} 5 a, n d u f b 1$, and $n d u f c 1$ are components of the electron transport chain in the mitochondrion. Therefore, we hypothesize that NTau may regulate the reduction-oxidation of $\mathrm{NADH}$ and thereby lead to the inhibition of HSC proliferation.

The pathogenesis of hepatic fibrosis involves the activation of HSCs. This procedure is accelerated by HSC proliferation and the progression of the cell cycle (29). NTau has been demonstrated to inhibit HSC proliferation and prevent HSCs in the G0/ G1 phase from entering the $\mathrm{S}$ and $\mathrm{G} 2 / \mathrm{M}$ phases by flow cytometric analysis, which suggests that NTau is able to modulate hepatic fibrosis. Functional clustering and GSEA analyses also showed that mitosis, and especially the $\mathrm{M}$ phase of mitotic cell cycle, was regulated by NTau treatment. Therefore, it was not only demonstrated that NTau was beneficial to hepatic fibrosis therapy, but valuable evidence to elucidate the underlying 
molecular mechanism by investigating responded genes and proteins was also provided.

It is worth noting that reduction-oxidation of NADH may play an important role in the protective effect of NTau against hepatic fibrosis, since GO functional clustering and GSEA analysis consistently came to the same results. The reduction-oxidation state is often used to describe the balance of $\mathrm{NAD}^{+} / \mathrm{NADH}$ and $\mathrm{NADP}^{+} / \mathrm{NADPH}$ in a biological system such as a cell (30). Oxidative stress is important in the pathogenesis of hepatic fibrosis, which is the result of deposition of excess ECM proteins produced by activated HSCs (6). NTau may be able to attenuate ROS production by modulating the balance of reduction-oxidation of NADH. Furthermore, the relationship between the reduction-oxidation of NADH and HSC proliferation or cell cycle regulation has been previously investigated. Zou et al (31) have demonstrated that ROS derived from NAD(P) $\mathrm{H}$ oxidases activated the phosphotidylinositol-3-kinase (PI3K)/ Akt pathway, thus promoting cellular proliferation in HSCs.

Taurine may be synthesized chemically or extracted from natural sources. However, NTau is superior to synthetic taurine in promoting HSC apoptosis (32). The advantage of the present study is reflected in the selection of NTau for investigation of its mechanism in regulating HSC apoptosis. To the best of our knowledge, the present study provided for the first time evidence concerning taurine-mediated transcriptional changes in HSCs by microarray analysis. Additionally, proteomic approaches were used to delineate protein expression changes in NTau-treated HSCs. These variations correspond to biological processes such as 'oxidant reaction' and 'mitosis process,' which promote HSC apoptosis. While these observations systematically investigated the underlying mechanism of NTau in inhibiting the activation of HSCs, our data provide strong support for the use of NTau as a potential therapy for hepatic fibrosis.

\section{Acknowledgements}

This study was supported by the National Natural Science Foundation of China (81160433) and the Guangxi Natural Science Foundation (2010GXNSFA013217). The authors thank Tiandiyang Biology Corporation and the SysBiomics Bioinformatics Co., Ltd. (Beijing, China), for their instructive help with data analysis.

\section{References}

1. Bataller R and Brenner DA: Liver fibrosis. J Clin Invest 115: 209-218, 2005.

2. Chen YX, Zhang XR, Xie WF and Li S: Effects of taurine on proliferation and apoptosis of hepatic stellate cells in vitro. Hepatobiliary Pancreat Dis Int 3: 106-109, 2004.

3. Wu J and Zern MA: Hepatic stellate cells: a target for the treatment of liver fibrosis. J Gastroenterol 35: 665-672, 2000.

4. Kerai MD, Waterfield CJ, Kenyon SH, Asker DS and Timbrell JA Taurine: protective properties against ethanol-induced hepatic steatosis and lipid peroxidation during chronic ethanol consumption in rats. Amino Acids 15: 53-76, 1998.

5. Balkan J, Dogru-Abbasoglu S, Kanbagli O, Cevikbas U, Aykac-Toker G and Uysal M: Taurine has a protective effect against thioacetamide-induced liver cirrhosis by decreasing oxidative stress. Hum Exp Toxicol 20: 251-254, 2001.

6. Refik Mas M, Comert B, Oncu K, Vural SA, Akay C, Tasci I, Ozkomur E, Serdar M, Mas N, Alcigir G and Yener N: The effect of taurine treatment on oxidative stress in experimental liver fibrosis. Hepatol Res 28: 207-215, 2004.
7. Ghandforoush-Sattari M and Mashayekhi S: Evaluation of taurine as a biomarker of liver damage in paracetamol poisoning. Eur J Pharmacol 581: 171-176, 2008.

8. Miyazaki T, Karube M, Matsuzaki Y, Ikegami T, Doy M, Tanaka N and Bouscarel B: Taurine inhibits oxidative damage and prevents fibrosis in carbon tetrachloride-induced hepatic fibrosis. J Hepatol 43: 117-125, 2005.

9. Bradford MM: A rapid and sensitive method for the quantitation of microgram quantities of protein utilizing the principle of protein-dye binding. Anal Biochem 72: 248-254, 1976.

10. Stoscheck CM: Quantitation of protein. Methods Enzymol 182: 50-68, 1990.

11. Ashburner M, Ball CA, Blake JA, et al: Gene ontology: tool for the unification of biology. The Gene Ontology Consortium. Nat Genet 25: 25-29, 2000.

12. Huang da W, Sherman BT and Lempicki RA: Systematic and integrative analysis of large gene lists using DAVID bioinformatics resources. Nat Protoc 4: 44-57, 2009.

13. Kanehisa M, Araki M, Goto S, Hattori M, Hirakawa M, Itoh M, Katayama T, Kawashima S, Okuda S, Tokimatsu T and Yamanishi Y: KEGG for linking genomes to life and the environment. Nucleic Acids Res 36: D480-D484, 2008.

14. Patterson TA, Lobenhofer EK, Fulmer-Smentek SB, et al: Performance comparison of one-color and two-color platforms within the MicroArray Quality Control (MAQC) project. Nat Biotechnol 24: 1140-1150, 2006.

15. Yang YH, Dudoit S, Luu P, Lin DM, Peng V, Ngai J and Speed TP: Normalization for cDNA microarray data: a robust composite method addressing single and multiple slide systematic variation. Nucleic Acids Res 30: e15, 2002.

16. Subramanian A, Tamayo P, Mootha VK, et al: Gene set enrichment analysis: a knowledge-based approach for interpreting genome-wide expression profiles. Proc Natl Acad Sci USA 102: 15545-15550, 2005.

17. Apweiler R, Bairoch A, Wu CH, et al: UniProt: the Universal Protein knowledgebase. Nucleic Acids Res 32: D115-D119, 2004.

18. Huang da W, Sherman BT, Tan Q, Collins JR, Alvord WG, Roayaei J, Stephens R, Baseler MW, Lane HC and Lempicki RA: The DAVID Gene Functional Classification Tool: a novel biological module-centric algorithm to functionally analyze large gene lists. Genome Biol 8: R183, 2007.

19. Kendler BS: Taurine: an overview of its role in preventive medicine. Prev Med 18: 79-100, 1989.

20. Lourenço R and Camilo ME: Taurine: a conditionally essential amino acid in humans? An overview in health and disease. Nutr Hosp 17: 262-270, 2002.

21. Chen YX: Protective action of taurine on ischemia-reperfusion liver injury in rats and its mechanism. Zhonghua Yi Xue Za Zhi 73: 276-279, 318-379, 1993 (In Chinese).

22. Friedman SL: Seminars in medicine of the Beth Israel Hospital, Boston. The cellular basis of hepatic fibrosis. Mechanisms and treatment strategies. N Engl J Med 328: 1828-1835, 1993.

23. Cotran RS, Kumar V and Collins T (eds): Robbins Pathologic Basis of Disease. 6th edition. WB Saunders Co., Philadelphia, 1998.

24. Mattson MP and Chan SL: Calcium orchestrates apoptosis. Nat Cell Biol 5: 1041-1043, 2003.

25. Simon HU, Haj-Yehia A and Levi-Schaffer F: Role of reactive oxygen species (ROS) in apoptosis induction. Apoptosis 5: 415-418, 2000.

26. Green DR and Reed JC: Mitochondria and apoptosis. Science 281: 1309-1312, 1998.

27. Camilleri-Broët S, Vanderwerff H, Caldwell E and Hockenbery D: Distinct alterations in mitochondrial mass and function characterize different models of apoptosis. Exp Cell Res 239: 277-292, 1998.

28. Shibanuma M, Mashimo J,Kuroki T and Nose K: Characterization of the TGF beta 1-inducible hic-5 gene that encodes a putative novel zinc finger protein and its possible involvement in cellular senescence. J Biol Chem 269: 26767-26774, 1994.

29. Wells RG: The role of matrix stiffness in hepatic stellate cell activation and liver fibrosis. J Clin Gastroenterol 39: S158-S161, 2005.

30. Ying W: $\mathrm{NAD}^{+} / \mathrm{NADH}$ and $\mathrm{NADP}^{+} / \mathrm{NADPH}$ in cellular functions and cell death: regulation and biological consequences. Antioxid Redox Signal 10: 179-206, 2008.

31. Zou CG, Gao SY, Zhao YS, Li SD, Cao XZ, Zhang Y and Zhang KQ: Homocysteine enhances cell proliferation in hepatic myofibroblastic stellate cells. J Mol Med (Berl) 87: 75-84, 2009.

32. Liang J, Deng X, Yang GY, Huang RB and Pang YS: Effect of natural taurine on serology and histology of hepatic fibrosis in rat. Journal of Guangxi Traditional Chinese Medical University 9: 3-5, 2006 (In Chinese). 